\title{
Communication Requirements of Emerging Cooperative Driving Systems
}

\author{
Elisabeth Uhlemann \\ Centre for Research on Embedded Systems \\ Halmstad University \\ Box 823, SE-301 18 Halmstad, Sweden \\ Email: Elisabeth.Uhlemann@hh.se
}

\begin{abstract}
Traffic safety applications relying on cooperative systems are currently being considered by several research projects worldwide. An important question is if existing wireless technologies can meet the communication requirements from this emerging field of applications? Part of the answer to this question is that the communication requirements depend on what is actually communicated and how this information is used by and presented to the driver. The data traffic from realizations based on "cooperative awareness" or on "hazard warnings" are very different. This article discusses the communication requirements of some typical traffic safety applications and how these requirements are affected by different realizations.
\end{abstract}

\section{INTRODUCTION}

Cooperative systems using wireless vehicular communications to complement intelligent transport systems (ITS) currently receive a great deal of attention worldwide, [1,2]. One of the main motivations is the possibility to reduce traffic accidents and human injuries by introducing traffic safety applications based on vehicular communications. Another is to reduce congestion, travel-time and pollution through traffic efficiency applications. Finally, other types of services may be offered in order to facilitate system introduction and provide sustainable business and operation models. Thus, mainly three types of application are considered: traffic safety, traffic efficiency and value-added services. The main focus of this article is traffic safety applications since the communication requirements of these applications notably differ from those of most existing applications relying on wireless communications.

The basic idea of traffic safety applications is to enhance the situation awareness of the driver such that the time to react to dangerous events is increased. Already today there are several examples of traffic safety applications, such as forward collision warning and blind spot detection, which do not use communications, but merely different types of sensors (radar, cameras, accelerometers, etc). Whenever a local sensor detects a hazard, the driver is notified. By introducing communications into these types of applications, they can be enhanced. For example, forward collision warning can be enhanced by information communicated from distant road signs or fellow road users braking beyond line of sight of the driver. The application blind spot detection can be enhanced by information transmitted from the vehicle located in the blind spot (e.g., weight, bumper height, number of passengers, dangerous

This work was funded in part by the Swedish Governmental Agency for Innovation Systems, Vinnova, through the VINNMER program, www.vinnova.se goods). Further, new applications can be developed that specifically benefit from communication since traditional sensors are insufficient, e.g., intersection collision warning.

An important question is if existing wireless technologies can be used for this specific field of applications. For example, what functionalities are needed and expected of the applications in terms of communication system response times and reliability? Do these functionalities require $100 \%$ penetration? Are the systems scalable and can they be made sufficiently reliable and robust? Part of the answer to these questions is that applications such as the above can be implemented in several different ways. Depending on what kind of information that is exchanged, how it is used and presented to the driver, and what happens during temporary communication link failures, the resulting requirements are very different.

\section{DATA TRAFFIC MODELS}

The traffic safety applications forward collision warning, blind spot detection and intersection collision warning mentioned above could be implemented either as a "hazard warnings" system or as a "cooperative awareness" system [3]. There is also a third realization referred to here as "autonomous cooperative driving". Depending on realization different types of data traffic are generated, which in turn have different communication requirements.

\section{A. Hazard Warnings}

Hazard warnings are communicated only in the event of a hazard. When an incident arises, the application triggers a message containing information about the hazard itself. This kind of event-driven messaging requires very high reliability since the data is critical for traffic safety. A low delay is also important since we want to increase the driver awareness horizon by notify other drivers about an upcoming event as early as possible. Since the hazard warnings are typically broadcasted, the reliability can be increased through periodically repeated broadcasting until the event has occurred or no longer is valid. This realization is useful also at moderate penetration rates since it can be implemented as an enhancement of existing sensors and complement already existing applications. The hazard warnings can be propagated a certain distance by e.g., vehicles in opposite direction using so-called geocasting capabilities, but most warnings are needed only when the hazard is imminent and therefore they typically have a short time and range of interest. 


\section{B. Cooperative Awareness}

If all vehicles are equipped with communication devices and regularly broadcast their positions, speed and directions, a socalled Local Dynamic Map (LDM) can be constructed, [3]. A LDM would also contain static map information and temporary information about e.g., road conditions communicated from selected road-side units. The cooperative awareness messages are the foundation of many applications $[4,5]$. This realization implies that time-triggered messages are broadcasted periodically by all vehicles. Since the messages are repeated periodically and do not signal imminent hazard the requirement on reliability is moderate. The LDM based applications can predict trajectories on vehicles depending on previously received messages even if some messages are lost. The LDM can be used to predict dangerous situations before they actually occur. For example, "it will be a collision within four seconds if all vehicles maintain the same speed and direction". The system can thereby extend the horizon of awareness of the driver. In order to avoid a system with invisible, mute vehicles, a high penetration is likely needed. However, also at lower penetration rates cooperative awareness messages could be used as application enhancement and driver guidance. Since messages are broadcasted by all vehicles regularly, there is limited need to retransmit them or let them propagate beyond communication range. Instead, more effort should be put on selection of appropriate communication ranges since the needs are likely different in urban areas as compared to inter-urban.

\section{Autonomous Cooperative Driving}

The autonomous cooperative driving concept is the long-term goal of vehicular networks, where the driver has an autopilot and can just lean back and relax. Not only is this system required to warn, predict or advice - but also to act. Consequently, an autonomous cooperate driving system would typically be using both periodic time-triggered and event-driven messages, requiring some sort of service differentiation between the two types of messages, and very likely close to $100 \%$ penetration will be needed.

\section{DATA CONTENTS AND USE}

An issue that greatly influences the communication requirements is the selected level of autonomy. The more reliable the communication system is, the higher autonomy can be given to the application. With the introduction of communication between vehicles, the received information will initially be used to inform the driver, then warn and advice, Fig. 1, followed by guide and finally steer, ending up with a cooperative autonomous driving system as penetration rates increase coupled with increasing requirements on reliability.

The control loop that is using the communicated data consists of four steps: observation, evaluation, decision and action. The received data typically corresponds to an observation. However, the communicated messages could either be raw data (observation) or some level of refined data (evaluation) or even the actual action of another vehicle. The latter is likely to require a higher penetration rate such that decisions

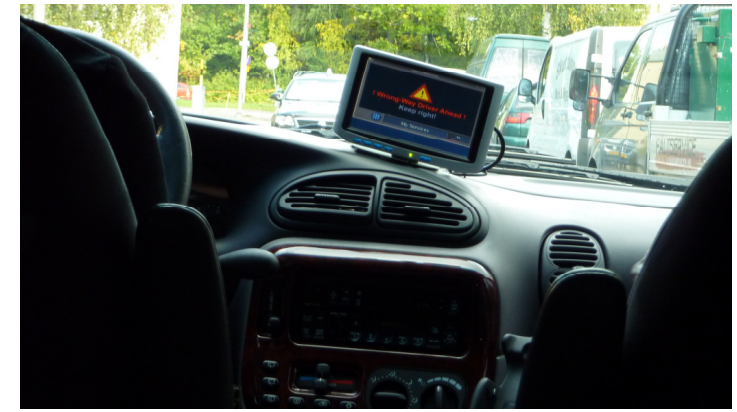

Figure 1. Example of user interface from the CVIS project: Warning: "Wrong Way Driver Ahead!". Advice: "Keep Right."

are made based on the same information. However, transmitting processed data is likely to required less bandwidth. Therefore, when penetration increases, more and more processed data could be transmitted to compensate for the increase in required collective bandwidth.

\section{CONCLUSIONS}

Traffic safety applications need reliable, predictable, lowdelay, wireless communications. These requirements differ from those of most existing applications relying on wireless communications, as reliability, predictability and low-delay are required jointly. The high speed of the vehicles and the harsh, dynamic communication environment makes is extremely difficult to meet the requirements. However, whether it is possible for a specific wireless technology to fulfill the requirements depends not only on the application in question, but also on how it is implemented. Rather than discussing specific numerical values of the communications requirements, this paper deals with different ways to implement traffic safety applications and how these implementations affect the requirements. It can be concluded that traffic safety applications should be designed and evaluated by application and communication specialists jointly, since no communication system can ever be made error free and thus all applications must be designed to handed transient errors.

\section{REFERENCES}

[1] L. Strandén, E. Uhlemann, and E. G. Ström, "State of the art survey of wireless vehicular communication projects," in Proc. 15th World Congress on ITS, New York City, NY, Nov. 2008.

[2] P. Papadimitratos, et al., "Vehicular communication systems: Enabling technologies, applications, and future outlook on intelligent transportation," IEEE Communications Magazine, vol. 47, no. 11, pp. 84-95, Nov. 2009.

[3] T. Kosch, et al., "Communication architecture for cooperative systems in Europe," IEEE Communications Magazine, vol. 47, no. 5, pp. 116-125, May 2009.

[4] ETSI TC ITS, Basic set of applications; Functional Requirements, Working draft TS102 637-1, Jun. 2010.

[5] IEEE 802.11p, Part 11: Wireless LAN MAC and PHY specifications: Amendment 7: Wireless Access in Vehicular Environment, Jul. 2010. 